\title{
Biodegradabilidad de lixiviados procedentes de un relleno sanitario utilizando un sistema de lodos activados flujo pistón
}

\author{
The biodegradability of leachates from a landfill using an activated \\ sludge processes plug flow reactor system
}

\section{Biodegradabilidade de lixiviados de um aterro usando um sistema de lodos ativados de fluxo de pistão}

\author{
Jenny A. Rosas-Vargas ${ }^{1}$, Ing. Biot, Esp, MSc; Jacipt A. Ramón-Valencia ${ }^{2}$, IQ, MSc, PhD \\ 1 Grupo de Investigaciones Ambientales Agua, Aire y Suelo (GIAAS). Universidad de Pamplona, Pamplona, Colombia \\ 2 Docente Investigador, Facultad de Ingenierías y Arquitectura, Universidad de Pamplona, Pamplona, Colombia \\ Email: rosasjenny2007@hotmail.com
}

Recibido: 04 de septiembre de $2018 \quad$ Aceptado: 01 de agosto de 2020

\begin{abstract}
Resumen
En esta investigación se desarrolló un Reactor Flujo Pistón (RFP) de lodos activados, que fue empleado para determinar el Tiempo de Retención Celular (TRC) óptimo para tratar cargas altas de lixiviado y estimar el comportamiento cinético del sistema con el fin que sirva de base para proyecciones de escalamiento y diseño de reactor de este tipo a escala real. Para ello se realizó el diseño, la construcción y puesta en marcha del RFP a nivel de laboratorio (capacidad de 50L) en condiciones de operación continua con recirculación de lodo y se utilizó como sustrato lixiviado procedente del relleno sanitario El Guayabal de la ciudad de Cúcuta. Se determinó como parámetros de control cuatro TRC diferentes entre 5 y 20 días diferenciada por etapas. Se evaluaron las variables de pH, Temperatura, Oxígeno disuelto y Sólidos Suspendidos Volátiles en el Licor Mixto (SSVLM). En cuanto al control y monitoreo de la eficiencia de remoción se escogió la Demanda Química de Oxígeno (DQO) como parámetro para medir la degradación de materia orgánica, dado que este parámetro a diferencia de la Demanda Bioquímica de Oxígeno (DBO5) puede ser calculado en un menor tiempo. El sistema biológico RFP alcanzó estabilizarse en un período de operación entre 20 y 25 días con remociones promedio mayores a $92 \%$ para TRC de 15 y 20 días mientras que TRC menores reportaron remociones promedio por debajo del 86\%. Así mismo TRC de 15 permitió un Factor de carga (F/M) óptima promedio de 1.1 KgDQO/KgSSV.d. Las constantes cinéticas obtenidas en esta investigación, representativas para un sistema de RFP operado a escala laboratorio para remoción de materia orgánica carbonacea en lixiviados fueron: $\mathrm{KS}=836.5 \mathrm{mg} / \mathrm{L} \mathrm{DQO}, \mathrm{k}=4.8123 \mathrm{~d} 1, \mathrm{Y}=0.089 \mathrm{mg} \mathrm{SSVLM} / \mathrm{mg}$ DQO y $\mathrm{kd}=0.018 \mathrm{~d}-1$, ajustados por regresión al modelo matemático de Lawrence y McCarty al poseer una correlación de 0.97 entre los datos experimentales obtenidos y el modelo y amplia aplicación en el campo de diseño de reactores de lodos activados.
\end{abstract}

Palabras clave: factor de carga, modelo matemático, parámetros cinéticos, reactor flujo pistón, recirculación de lodos, tiempo de residencia celular. 


\begin{abstract}
An activated sludge processes (ASP) plug flow reactor (PFR) was developed in this research and used for determining optimal cell residence time (CRT) for treating high leachate loads and estimating the system's kinetic behaviour as a basis for scaling projections and this type of reactor design at full-scale. The RFP was designed, built and put into operation in the laboratory (50L capacity) involving continuous operation conditions with sludge recirculation; leached substrate from the city of Cúcuta's El Guayabal landfill was used. Four 5-day CRTs (ranging from 5 to 20 days) were used as control parameters. Variables were evaluated regarding $\mathrm{pH}$, temperature, dissolved oxygen and mixed liquor volatile suspended solids (MLVSS). Regarding removal efficiency control and monitoring, chemical oxygen demand (COD) was chosen as a parameter for measuring organic matter degradation since COD (unlike biochemical oxygen demand (BOD5)) can be calculated in a shorter time. The RFP biological system became stabilised in a 20- to 25-day operating period, having greater than $92 \%$ average removal for 15- and 20-day CRTs, whilst lower CRTs reported lower than 86\% average removal. A 15 -day TRC gave an average $0.26 \mathrm{kgDQO} / \mathrm{kgSSV} . \mathrm{d}$ optimal load factor $(\mathrm{F} / \mathrm{M})$. The representative kinetic constants obtained in this research for a laboratory-scale PFR system for the removal of carbonaceous organic matter in leachates were KS $=836 \mathrm{mg} C O D / \mathrm{l}$, $\mathrm{k}=4.8123 \mathrm{~d} 1, \mathrm{Y}=0.089 \mathrm{mg}$ SSVLM / mg COD and $\mathrm{kd}=0.018 \mathrm{~d}-1$, adjusted by regression using Lawrence and McCarty's mathematical model (having 0.97 correlation between the experimental data and the model). Such model should have broad application in the field of ASP-type PFRs.
\end{abstract}

Keywords: load factor, mathematical model, kinetic parameter, plug flow reactor, sludge recirculation, cell residence time.

\begin{abstract}
Resumo
Nesta pesquisa, um Reator de lodo ativado de Fluxo de Pistão (RFP) foi desenvolvido para determinar o Tempo de Retenção Celular (CRT) ideal para tratar altas cargas de lixiviado e estimar o comportamento cinético do sistema, a fim de servir de base para projetar o reator em escala real. Para isso, foram realizados RFP em nível de laboratório (capacidade de 50L) em condições de operação contínua com recirculação de lodo e foi utilizada como substrato lixiviado do aterro El Guayabal na cidade de Cúcuta. Usando quatro diferentes CRTs entre 5 e 20 dias como fatores de bloqueio. Foram avaliadas as variáveis pH, Temperatura, Oxigênio Dissolvido e Sólidos Suspensos Voláteis no Licor Misto (SSVLM). Em relação ao controle e monitoramento da eficiência de remoção, a Demanda Química de Oxigênio (DQO) foi escolhida como parâmetro para medir a degradação da matéria orgânica, uma vez que este parâmetro, ao contrário da Demanda Bioquímica de Oxigênio (DBO5), pode ser calculado em um tempo mais curto. O sistema biológico da RFP se estabilizou em um período de operação entre 20 e 25 dias com remoções médias superiores a 92\% para TRC de 15 e 20 dias, enquanto TRC menores mostraram remoções médias abaixo de 86\%. Da mesma forma, TRC de 15 dias permitiu um fator de carga ideal médio ( $/ \mathrm{M}$ ) de 0,26 KgDQO / d. As constantes cinéticas obtidas nesta pesquisa, representativas de um sistema RFP operado em escala de laboratório para remoção de matéria orgânica carbonácea em lixiviados foram: KS = 836mg COD / I, $\mathrm{k}=4,8123 \mathrm{~d} 1, \mathrm{Y}=0,089 \mathrm{mg}$ SSVLM / mg COD e $\mathrm{kd}=0,018 \mathrm{~d}$-1, ajustado por regressão ao modelo matemático de Lawrence e McCarty, tendo uma correlação de 0,97 entre os dados experimentais obtidos e o modelo e ampla aplicação na área de projeto de reatores de lodo ativado.
\end{abstract}

Palavras-chave: fator de carga, modelo matemático, parâmetros cinéticos, reator de fluxo em pistão, recirculação de lodo, tempo de residência da célula

\section{Introducción}

Los lixiviados generados en un relleno sanitario son efluentes de composición compleja y variable en el tiempo al ser influenciados por múltiples factores entre los que cabe destacar la composición de la basura, la edad del relleno, la solubilidad de los desechos y los procesos de conversión microbiológica y quími$\mathrm{ca}$, por lo cual se hace necesaria una secuencia de procesos de tratamiento, cada uno de ellos actuando sobre determinado(s) componente(s) del lixiviado para poder bajar las cargas contaminantes a los límites ambientales para vertimiento (Chávez, 2011).

Existen varios antecedentes de tratamiento biológico de lixiviados, que van desde sistemas aerobios como lodos activados convencional y en oxidación exten- dida, seguido por lagunas artificiales, hasta sistemas anaerobios entre los más experimentados están el reactor FAFA (Reactor Anaerobio de Flujo Ascendente) y reactor flujo pistón en condición anóxica (Giraldo, 2011).

Los lixiviados generados en el relleno sanitario "El Guayabal" son variables en cuanto a los caudales y concentraciones de materia orgánica como resultado de la incidencia de las precipitaciones en la zona de disposición final de los residuos (Álvarez y Suárez, 2006). Actualmente, estos son tratados mediante procesos físicos de evaporación y sedimentación en un complejo de tres (3) piscinas revestidas de geomembrana, sin embargo, dadas las altas cargas contaminantes no permiten su reducción a los límites de vertimientos exigidos en la normatividad ambiental. 
El Reactor Flujo Pistón (RFP) es un método ampliamente utilizado para los sistemas de lodos activados, el cual se basa en el régimen de flujo axial que permite la mezcla de las partículas de manera perpendicular al flujo reduciendo gradualmente la carga orgánica sin ninguna mezcla en la dirección longitudinal (Vargas y Torres, 2008). Esta variación permite la disminución del volumen del reactor, del lodo recirculado y de la cantidad de aire requerida a lo largo del tanque (Ramírez, 2008). El RFP ha sido utilizado a escala piloto para diferentes tipos de efluentes desde aguas residuales domésticas hasta aguas residuales de la industria textil, papelera, frigorífica, de curtiembres. No obstante, para el tratamiento de lixiviados solo se encontraron algunas experiencias a escala laboratorio en sistemas anóxicos, por lo que el diseño se realiza con base en los valores típicos para aguas residuales, siendo los ixiviados muy diferentes en cuanto a las altas concentraciones de materia orgánica y la presencia de compuestos que interfieren en la actividad microbiológica de biodegradación, lo cual puede repercutir en bajas eficiencias de remoción en los sistemas de tratamiento.

Por lo anterior, este trabajo investigativo busca determinar de forma experimental los parámetros cinéticos específicos para lixiviados que caractericen el funcionamiento del sistema en reactores tipo Flujo Pistón, y el rango óptimo de TRC como factores importantes de diseño y operación con el propósito de facilitar en el campo ingenieril el uso de valores más precisos in ne- cesidad de realizar montajes experimentales para cada condición o escalamiento a nivel de laboratorio.

\section{Materiales y métodos}

Diseño del modelo físico Reactor Flujo Pistón (RFP) Para el diseño del reactor piloto se aplicaron las ecuaciones para sistemas de lodos activados en reactor flujo pistón, presentadas en la Tabla 1.

Construcción y puesta en marcha del RFP. El modelo físico se representa esquemáticamente en la figura 1 y consta básicamente de un flujo de alimentación de lixiviados (a) que entra a un tanque rectangular de vidrio con dimensiones 0,60 $\mathrm{m}$ de largo, 0,25 $\mathrm{m}$ de ancho y $0,35 \mathrm{~m}$ de altura total, con un volumen útil de $50 \mathrm{~L}$, de los cuales 37,5 L corresponden al reactor o tanque de aeración (b) y 10,5 L al sedimentador (c). La aeración se realizó mediante difusión a través de una manguera de silicona de $4 \mathrm{~mm}$ de diámetro con orificios, insertada en el fondo de la cámara de aireación y conectada a 2 bombas aireadoras tipo mouse marca POWER LIFE P-500 con doble salida y 2,7 W de potencia.

Una vez tratados los lixiviados, se clarifican en el sedimentador y pasan al flujo de salida (d) a través de una abertura transversal en la parte superior del mismo, controlando que sea igual al caudal de entrada para conservar condiciones estacionarias en el sistema. Los lodos que se decantan pasan a una línea de recirculación (e), que consiste en una manguera de poliuretano y se envían al reactor nuevamente a una Tasa de Recirculación regulada, impulsados por una bomba

Tabla 1. Ecuaciones de diseño para sistemas de Lodos Activados tipo PFR.

\begin{tabular}{|l|c|}
\hline \multicolumn{1}{|c|}{ Parámetro } & Ecuación \\
\hline Eficiencia & $E=\frac{S o-S}{S o}=* 100$ \\
\hline Concentración de sustrato en el efluente & $S=\frac{K m(1+K d * \theta c)}{\theta c(Y * k-K d)-1}$ \\
\hline Biomasa en el Reactor & $X=\frac{\theta c Y(S o-S)}{\theta(1+K e \theta c)}=$ \\
\hline Producción de Lodos & $P x / s=\frac{Y}{(1+(K d * \theta c)} * Q(S o-S) / 1000$ \\
\hline Tiempo de Retención de Lodos $(\mathrm{V} / \mathrm{Q})$ & $\boldsymbol{\theta}=\boldsymbol{\theta} \boldsymbol{c}(1+R-R(X r / X))$ \\
\hline Edad de Lodos & $X=\frac{\theta c Y(S o-S}{\theta(1+K e \theta c)}$ \\
\hline
\end{tabular}

Fuente: Ramón et al., (2016). Software de simulación para el diseño de reactores de lodos activados. Revista Colombiana de Tecnologías de Avanzada, 2(28), 77-81. 
peristáltica con potencia máxima de $75 \mathrm{l} / \mathrm{h}$. Por último, se instaló en la parte inferior del reactor llaves de paso de $1 / 2$ pulgada para el flujo de purga $(\mathrm{f})$, con el objeto de regular el tiempo de residencia celular del sistema.

En la figura 2, se muestra detalladamente el montaje experimental del RFP de lodos activados en condiciones de operación continua con reciclo de lodos. Cabe resaltar que dos veces por semana de operación, se cargó el tanque de alimentación con muestras compuestas del afluente con el fin de mantener constantes los caudales según el tiempo de retención hidráulico escogido en la semana y así evitar que parámetros como la DQO cambiaran con el paso del tiempo.

\section{Desarrollo experimental}

El parámetro seleccionado para calcular la cantidad de sustrato en el sistema (S), fue la DQO. Se escogió este parámetro debido a que puede ser calculado en un tiempo promedio de 3 horas, tiempo que es significativamente más corto que los 5 días requeridos para el cálculo la DBO, tomada a la entrada (So) y a la salida del sistema ( $\mathrm{S}$ ).

El sistema fue operado en función del tiempo de retención hidráulica y TRC. El tiempo de retención hidráulico se determinó teniendo en cuenta el caudal mínimo arrojado por la bomba de alimentación utilizando la ecuación 1.

$$
\theta=\frac{V}{Q}
$$

Ecuación 1

En la Tabla 2 se muestran los tiempos de retención hidráulico y de retención celular con los que fue evaluado el sistema para cada una de las cuatro etapas, como también los caudales suministrados a la entrada del sistema. La razón de variar los tiempos de retención hidráulico y de retención celular (correspondiente a cada etapa) se debe a que para la determinación de los coeficientes cinéticos, es necesario obtener datos de SSVLM y DQO en mínimo cuatro tiempos diferentes.

Para controlar los valores de TRC medio se realizó purga de lodos directamente del reactor aplicando la ecuación 2, aunque durante el arranque del sistema no se recirculó lodo puesto que el objetivo era el aumento de biomasa dentro del reactor.

$$
Q w=\frac{V r X}{\theta c X r}
$$

Ecuación 2

Donde:

$\mathrm{V} \quad=$ Volumen reactor, en $\mathrm{L}$

$\mathrm{X}=$ concentración de biomasa en el reactor, en $\mathrm{mgSSV} / \mathrm{L}$

$\mathrm{X}_{\mathrm{r}}=$ Lodo recirculado, en $\mathrm{mgSSV} / \mathrm{L}$

$\mathrm{Q}_{\mathrm{w}}=$ Caudal de desecho, en $\mathrm{L} / \mathrm{d}$.

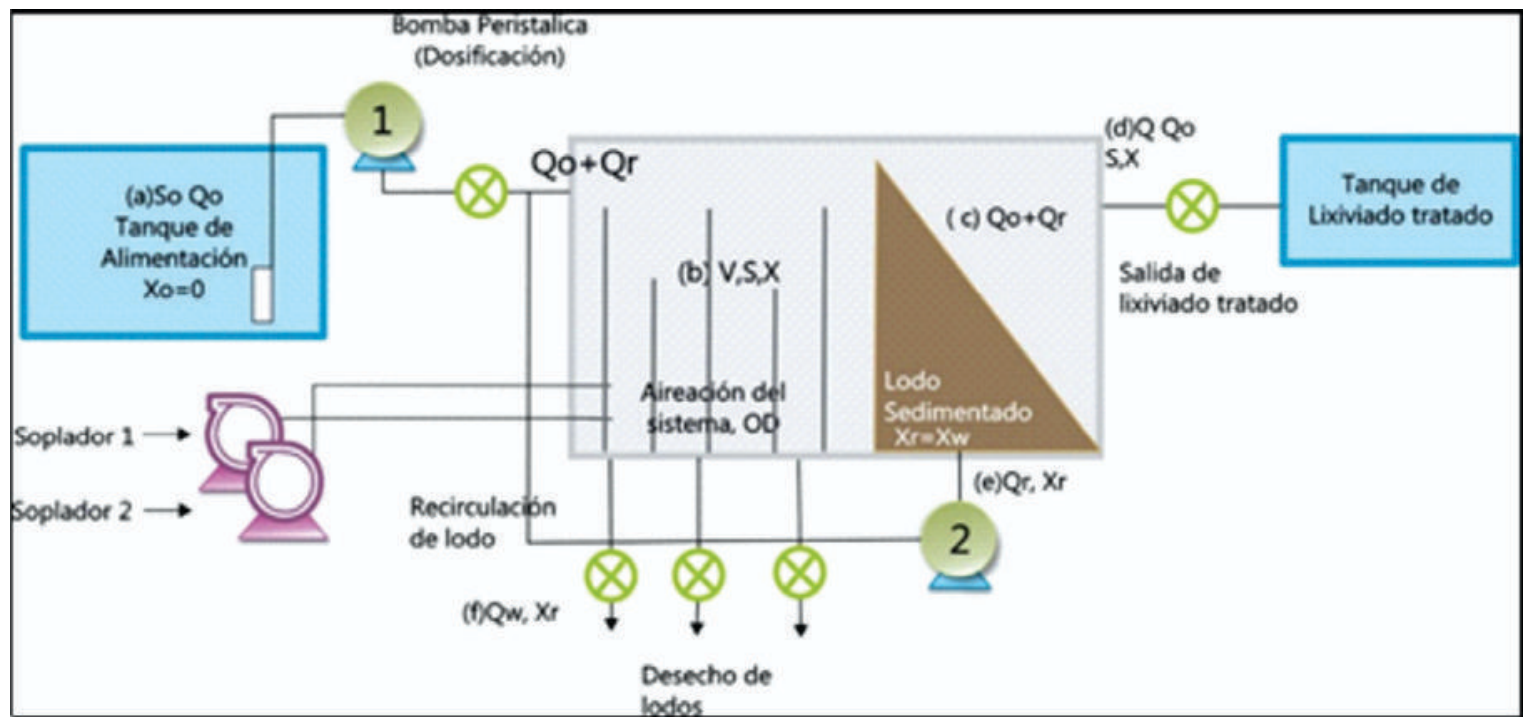

Figura 1. Modelo físico del Reactor Flujo Pistón de lodos activados con recirculación.

Donde,

$\mathrm{Q} \quad=$ caudal de alimentación

$\mathrm{Q}_{\mathrm{R}}=$ caudal de recirculación

$\mathrm{QW}=$ caudal de desecho
$\mathrm{V} \quad=$ volumen reactor

$\mathrm{S} \quad=$ concentración de DQO

$\mathrm{X}=$ concentración de biomasa en el reactor

$X_{R}=$ concentración de biomasa de recirculación 


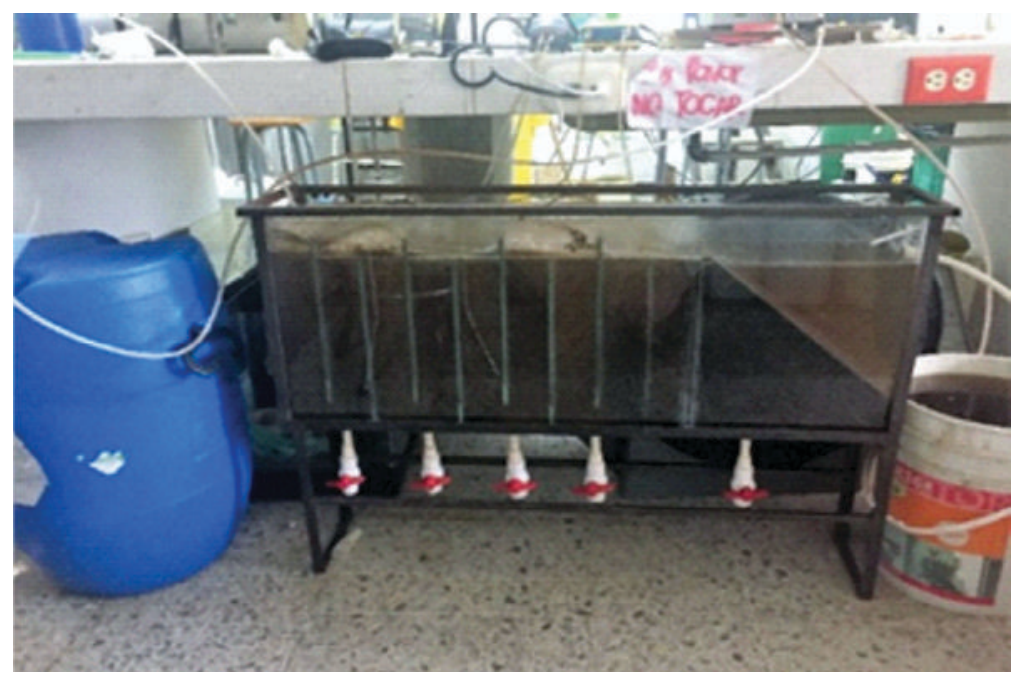

Figura 2. Montaje experimental del reactor flujo pistón (RFP)

Tabla 2. Criterios de operación del sistema de lodos activados RFP

\begin{tabular}{|c|c|c|c|}
\hline Etapa & $\boldsymbol{\theta}$ (horas) & $\begin{array}{c}\text { TRC } \\
\text { (días) }\end{array}$ & Caudal Q (L/h) \\
\hline 1 & 18,52 & 20 & 2,69978402 \\
\hline 2 & 13,44 & 15 & 3,7202381 \\
\hline 3 & 8,77 & 10 & 5,70125428 \\
\hline 4 & 3,99 & 5 & 12,5313283 \\
\hline
\end{tabular}

En cada etapa se realizó muestreo y análisis de los parámetros descritos en la Tabla 3, siguiendo las metodologías propuestas por APHA (2005) para aguas residuales.

El tiempo proyectado para la operación del sistema fue entre 3 a 7 semanas para cada etapa, según el tiempo de residencia celular hasta lograr estabilidad en el sistema, tomando como criterio de estabilidad una variación en la remoción de DQO igual o inferior al 10\%, la cual se determinó aplicando la ecuación 3 .

$$
\frac{F}{M}=\frac{S o \cdot Q}{V \cdot X r}
$$

Ecuación 3

Donde:

$S$ representa la concentración de materia orgánica en términos de mgDQO/L analizada a la entrada (SO) y salida (S) del RFP.

Adicionalmente, se calculó (F/M) como parámetro de control utilizando la ecuación 4.

$$
Q w=\frac{V r X}{\theta c X r}
$$

Ecuación 4

Donde:

So $=$ Demanda bioquímica de oxígeno $(\mathrm{DBO} 5)$ del agua a tratar, $\mathrm{g} / \mathrm{m}^{3}$.

$\mathrm{Q}=$ Caudal del agua residual a tratar, $\mathrm{m}^{3} /$ día .

$\mathrm{V}=$ Volumen del tanque de aeración, $\mathrm{m}^{3}$.

$X_{r}=$ Concentración de sólidos suspendidos volátiles en el tanque de aeración, g/ $/ \mathrm{m}^{3}$.

Determinación de constantes cinéticas y estequiométricas del sistema reactor flujo pistón de lodos activados. Logradas las condiciones de estabilización del reactor se comenzó el tratamiento del líquido residual. Se registraron los valores promedio de Demanda Química de Oxígeno (DQO) y Sólidos Suspendidos Volátiles en el licor mezcla (SSVLM) a través del tiempo y se ajustó la curva que mejor se adaptó empleando las ecuaciones cinéticas descritas en la Tabla 4. Los valores de los coeficientes cinéticos fueron estimados por medio de análisis de regresión de los datos experimentales. En este estudio se determinó el coeficiente de velocidad media (KS), la tasa máxima de degradación de sustrato (k), el coeficiente de producción de biomasa (Y) y la velocidad de decaimiento o muerte de microorganismos ( $k d)$.

\section{Resultados y discusiones}

Comportamiento de las variables operacionales. En las Tablas 5 y 6 se muestra los valores promedio de las variables operacionales como temperatura, $\mathrm{pH}$ y Oxígeno Disuelto, así como la eficiencia de remoción materia orgánica y la relación de alimento biomasa o factor de carga (F/M) para cada etapa. 
Tabla 3. Parámetros de control

\begin{tabular}{|c|c|c|c|c|c|c|}
\hline Parámetro & Unidad & Frecuencia & Afluente & $\begin{array}{c}\text { Reactor } \\
\text { Biológico }\end{array}$ & Efluente & Lodo de Purga \\
\hline $\mathrm{T}$ & ${ }^{\circ} \mathrm{C}$ & Diario & $\mathrm{X}$ & $\mathrm{X}$ & & \\
\hline $\mathrm{pH}$ & & Diario & $\mathrm{X}$ & $\mathrm{X}$ & & \\
\hline $\mathrm{OD}$ & $\mathrm{mg} / \mathrm{L} \mathrm{O} 2$ & Diario & & $\mathrm{X}$ & & \\
\hline $\mathrm{S}$ & $\mathrm{mg} / \mathrm{l} \mathrm{DQO}$ & 3 veces por semana & $\mathrm{X}$ & & $\mathrm{X}$ & \\
\hline $\mathrm{X}$ & $\mathrm{mg} / \mathrm{SSSV}$ & 3 veces por semana & & $X$ & & $X$ \\
\hline & $\mathrm{mg} / \mathrm{l} \mathrm{SST}$ & 3 veces por semana & & $X$ & & $X$ \\
\hline
\end{tabular}

Tabla 4. Ecuaciones cinéticas para sistemas de lodos activados.

\begin{tabular}{|c|c|c|c|}
\hline Coeficiente Cinético & Unidades & Modelo Matemático & Autor \\
\hline K & $\mathrm{mgSSV} / \mathrm{L}$ & $\frac{S o-S}{x \theta} v S S$ & Eckenfelder \\
\hline $\mathrm{KL}$ & $d-1$ & $\frac{S o-S}{\theta} v S S$ & Mc Kinney \\
\hline $\mathrm{Km}, \mathrm{k}$ & $\mathrm{mg} / \mathrm{L}, \mathrm{d}-1$ & $(\mathrm{X} \theta) /(\mathrm{So}-\mathrm{S})$ vs $1 / \mathrm{S}$ & Lawrence y McCarty \\
\hline $\mathrm{Ks}, \mathrm{k}$ & $\mathrm{mg} / \mathrm{L}, \mathrm{d}-1$ & $\frac{X \theta}{S O-S} v S \frac{1}{S}$ & Orozco \\
\hline $\mathrm{Kd}, \mathrm{Y}$ & $\mathrm{d}-1, \mathrm{mgSSV} / \mathrm{mgDBO}$ & $\frac{1}{\theta c} v s \frac{Y(S o-S)}{X \theta}-K d$ & Metcalf y Eddy \\
\hline
\end{tabular}

Fuente: Modificado de Orozco Jaramillo, A. (2014). Bioingeniería de aguas residuales. Teoría y diseño. Editorial Acodal. $2^{\circ}$ edición. Bogotá, Colombia. p 136-150.

Los valores reportados indican un $\mathrm{pH}$ ligeramente alcalino con promedios que oscilaron entre 8.2 y 8.4 , siendo 8.24 el valor más reportado (Mediana estadística). (Arguedas, 2013) señala un rango de pH para sistemas aerobios entre 6.5 y 8 para la remoción de contaminantes biodegradables, valores por fuera del rango pueden inhibir el crecimiento bacteriano. Aunque el valor se encuentra por fuera del rango es aceptable teniendo en cuenta que los lixiviados se caracteriza por presentar $\mathrm{pH}$ por encima de 8.5 unidades, igualmente autores reconocen cierto riesgo en la utilización generalizada de estos parámetros ya que los valores llegan a variar en amplios rangos debido a la influencia por condiciones específicas del efluente, y debe por tanto, ser determinados para cada tipo de agua residual en particular en diferentes PTAR (Espinosa et al., 2012).

La variable Temperatura osciló dentro de un rango de 18 a $23^{\circ} \mathrm{C}$, que en relación a la temperatura óptima de un sistema de lodos activados $\left(25\right.$ a $\left.35^{\circ} \mathrm{C}\right)$, propuesto

Tabla 5. Valores promedio de las variables operacionales del reactor flujo pistón RFP

\begin{tabular}{|c|c|c|c|c|c|c|c|c|c|c|c|c|c|c|c|}
\hline \multirow{2}{*}{ Etapa } & \multicolumn{5}{|c|}{ Temperatura $\left({ }^{\circ} \mathrm{C}\right)$} & \multicolumn{5}{|c|}{ pH (Unidades) } & \multicolumn{5}{|c|}{ Oxígeno Disuelto (mg/L) } \\
\hline & Rango & $\bar{X}$ & Med & $\Sigma$ & $\mathbf{N}$ & Rango & $\bar{x}$ & Med & $\Sigma$ & N & Rango & $\bar{X}$ & Med & $\Sigma$ & $\mathrm{N}$ \\
\hline 1 & $18-22$ & 19,82 & 20 & 1,08 & 34 & $8,03-8,68$ & 8,29 & 8,24 & 0,17 & 27 & $4,23-5,78$ & 5,29 & 5,36 & 0,41 & 27 \\
\hline 2 & $18-22$ & 19,84 & 20 & 0,87 & 37 & $7,91-8,49$ & 8,25 & 8,29 & 0,14 & 30 & $4,75-5,65$ & 5,44 & 5,50 & 0,22 & 30 \\
\hline 3 & $18-22$ & 20,11 & 20 & 0,69 & 38 & 7,86-8,66 & 8,24 & 8,24 & 0,21 & 31 & $3,31-5,58$ & 4,42 & 4,52 & 0,55 & 31 \\
\hline 4 & $18-23$ & 19,83 & 20 & 1,28 & 48 & $8,13-8,71$ & 8,39 & 8,42 & 0,13 & 37 & $3,23-5,52$ & 4,31 & 4,32 & 0,49 & 37 \\
\hline
\end{tabular}

$X$ : media aritmética; Med: mediana; $\sigma$ : desviación estándar; $n$ : número de lecturas. 
por Metcalf y Eddy (2003), se aprecia que la temperatura al interior del reactor fue más baja; sin embargo, el lodo biológico presentó una remoción satisfactoria lo cual supone que la población microbiana del lodo inoculado ya se encontraba aclimatada para trabajar a estas temperaturas. En general, a temperaturas entre 20 y $45^{\circ} \mathrm{C}$ se alcanzan eficiencias de reducción de DQO en lixiviados entre el 70 al $80 \%$, dependiendo de la edad del lixiviado y la relación DBO5 /DQO (Torres et al., 2014).

En cuanto al Oxígeno Disuelto (OD), las concentraciones promedio en las 4 etapas estuvieron en el rango 4.31 y $5.58 \mathrm{mg} / \mathrm{L}$ en general valores altos que facilitan la oxidación de la materia orgánica. Giraldo y Restrepo (2003), señala que se debe asegurar una concentración entre 1 y 3 mg/L para controlar el desarrollo de organismos filamentosos ocasionado por sobreaireación, sin embargo, en otro estudio se encontró poca influencia al disminuir la concentración a $3 \mathrm{mg} / \mathrm{L}$ en cuanto a la calidad del floc formado (Rodriguez y Osorio, 2014). En general se estima que, la cantidad de oxígeno requerida oscila entre el $15-30 \%$ de la demanda química de oxígeno (DQO) soluble removida (Peng, 2003).

Con base a los datos presentados en la Tabla 6 se observa que hay una disminución en la remoción de materia orgánica a medida que disminuye el TRC, siendo Et4 (5 días) la que presentó menor eficiencia de remoción $(80.46 \%)$ con una concentración de biomasa en el reactor promedio de 1500 a 3000 mg/L SSV. Lo anterior se puede

atribuir a dos condiciones: en primer lugar, el factor de carga (F/M) estuvo muy por encima de lo recomendado 2.20KgDQO/KgSSV.d y segundo, las concentraciones de sustrato en la entrada (So) doblaron las concentraciones máximas de las aguas residuales domésticas que no logran superar 1200mg/L DQO. En un estudio realizado se encontró que, al reducir el tiempo de retención de los lodos de 15 a 7 días, mejoró la sedimentación, pero empeoró la calidad del efluente, debido a que al aumentar la relación F/M disminuye también la tasa de remoción de materia orgánica. Sin embargo, varios estudios concuerdan en que los reactores de flujo pistón favorecen un adecuado macro gradiente de la concentración del sustrato por sus compartimientos que garantice una alta tasa de consumo de sustrato y por ende se pueden manejar relaciones altas de (F/M). Rodríguez y Molina (2017).

Comportamiento de remoción de sustrato y de crecimiento celular en función del TRC. Las figuras 3 y 4 representan la influencia de los valores de TRC establecidos sobre la eficiencia de remoción de sustrato y la biomasa presente en el reactor (SSVLM).

En la figura 3 se aprecia que para las condiciones de operación de las etapas 1 y 2 se logró la estabilización del sistema a partir de los 20 días de operación, alcanzándose eficiencias superiores al 90\% (eficiencia promedio de 94.6\% para Et1 y 92.8\% para Et2). Para la etapa 3 aunque el sistema alcanzó regularse alrededor de 30 días de operación la eficiencia de remoción promedio no superó el 84\%; igualmente, la etapa 4 con tiempo de retención de 5 días presentó mayor inestabilidad y tardó 35 días para alcanzar el estado estacionario propio de sistemas continuos con un máximo de $80 \%$ de remoción. Comparando los valores de remoción obtenidos con los valores típicos de remoción para aguas residuales domésticas (superiores al 90\%) con TRC que oscila en un rango entre 5 y 15 días, se tiene que dicho rango no aplica como óptimo para aguas residuales con concentraciones de materia orgánica muy altas (superiores a 1600mg/L DQO), como el caso de lixiviados cuyas concentraciones por lo general oscila entre 600 a $12000 \mathrm{mg} / \mathrm{L}$ DQO (López y Sánchez, 2015).

De acuerdo con Rodríguez y Osorio (2014), tiempos cortos de retención celular no permite la producción

Tabla 6. Valores promedio de las variables de control en función del TRC.

\begin{tabular}{|c|c|c|c|c|c|c|c|c|}
\hline Etapa & $\begin{array}{c}\text { Tiempo de } \\
\text { operación } \\
\mathbf{d}\end{array}$ & $\begin{array}{c}\mathbf{N}^{\circ} \\
\text { Muestra }\end{array}$ & $\begin{array}{c}\text { So } \\
\mathbf{m g} / \mathbf{I} \\
\mathbf{D Q O}\end{array}$ & $\begin{array}{c}\mathbf{S} \\
\mathbf{m g} / \mathbf{I} \\
\mathbf{D Q O}\end{array}$ & $\begin{array}{c}\mathbf{X}(\mathbf{S S V}) \\
\mathbf{m g} / \mathbf{I}\end{array}$ & $\begin{array}{c}\mathbf{X}(\mathbf{S S T}) \\
\mathbf{m g} / \mathbf{I}\end{array}$ & $\begin{array}{c}\mathbf{F} / \mathbf{M} \\
\mathbf{d}-\mathbf{1}\end{array}$ & $\begin{array}{c}\text { Efciencia } \\
\mathbf{\%}\end{array}$ \\
\hline 1 & 34 & 16 & 2986.06 & 168.08 & 4695.6 & 5079.9 & 0.824 & 94.37 \\
\hline 2 & 45 & 17 & 2973.731667 & 212.60 & 3777.0 & 3796.2 & 1.111 & 92.85 \\
\hline 3 & 45 & 19 & 3003.15 & 477.45 & 2642.7 & 3535.1 & 2.260 & 84.10 \\
\hline 4 & 48 & 24 & 2979.75 & 582.23 & 1360.7 & 1912.7 & 2.204 & 80.46 \\
\hline
\end{tabular}

* Los valores registrados de las variables presentadas corresponden al promedio aritmético del total de muestras tomadas para cada etapa. 
de sustancias poliméricas extracelulares (SPE) responsables de la biodegradabilidad del sustrato lo que repercute en la calidad del efluente. Para tiempos de retención más prolongados se obtuvo mejores resultados de remoción (Et1 y Et2), considerándose TRC óptimo a partir de 15 días para reactores de lodos activos tipo flujo pistón.

En la figura 4 se aprecia que los TRC de 10 y 5 días (correspondientes a las Et 3 y Et4 respectivamente) no favorecieron el crecimiento celular, manteniéndose entre los 1000 y 2000 mg/L SSV. Von Sperling (2012) indica que, para que ocurra una correcta adsorción del sustrato en sistemas de estabilización por suspensión el valor recomendado de biomasa en el reactor es de 1500 a 3500 mg/L; la baja concentración de biomasa obtenida se puede atribuir a las altas concentraciones de materia orgánica del lixiviado, ya que factores de carga (F/M) superiores a $0.82 \mathrm{KgDQO} / \mathrm{KgSSV}$.d. pueden provocar shock tóxico en los microorganismos responsables de la degradación (Ramos, 2017) o pueden estimular el aumento de microorganismos filamentosos que provocan el arrastre de SSV en el efluente.
Determinación de constantes cinéticas y estequiométricas del sistema reactor flujo pistón de lodos activados. En las Tablas 6 y 7 se muestran los datos para el cálculo de las constantes cinéticas.

En la figura 5 se muestra las representaciones gráficas de las regresiones lineales aplicadas para cada uno de los modelos matemáticos asumidos.

De acuerdo con la figura 5, los datos obtenidos experimentalmente se ajustaron mayormente a los modelos matemáticos de Lawrence y McCarty y Orozco con una correlación de 0.97. De acuerdo con Romero (2004) algunos modelos como el descrito por Eckenfelder, pueden presentar limitaciones para sustratos con concentraciones muy altas, como la inhibición por sustrato, donde hay una disminución progresiva de la actividad a altas concentraciones, por lo tanto, son poco aplicables para sistemas de tratamiento en condiciones de abundancia (CA).

La figura 6 representa el comportamiento de generación de biomasa $(\mathrm{Y})$ y el decaimiento de la misma $(\mathrm{Kd})$ determinados a partir de los datos obtenidos experimentalmente.

Tabla 6. Cálculos de ecuaciones cinéticas para sistemas de lodos activados.

\begin{tabular}{|c|c|c|c|c|c|c|c|c|}
\hline Etapa & So-S (mg/L) & $\begin{array}{c}\mathbf{1} / \mathbf{S}(\mathbf{m g} / \\
\mathbf{L})-\mathbf{1}\end{array}$ & $\begin{array}{c}\mathbf{1} / \boldsymbol{\theta} \mathbf{c} \\
\mathbf{( d )}-\mathbf{1}\end{array}$ & $\mathbf{X} \boldsymbol{\theta}$ & $\begin{array}{c}\mathbf{X} \boldsymbol{\theta} / \mathbf{S o}-\mathbf{S} \\
\mathbf{( d )}\end{array}$ & $\begin{array}{c}\text { So-S } / \mathbf{X} \boldsymbol{\theta} \\
(\mathbf{d})-\mathbf{1}\end{array}$ & $\begin{array}{c}\text { So-S } / \boldsymbol{\theta} \\
(\mathbf{d})-\mathbf{1}\end{array}$ & $\mathbf{X} / \mathbf{S}$ \\
\hline 1 & 2817,980 & 0,0059 & 0,05 & 3623,515 & 1,285 & 0,777 & 3651,809 & 27,936 \\
\hline 2 & 2761,126 & 0,0047 & 0,06 & 2675,12 & 0,968 & 1,032 & 4930,583 & 22,468 \\
\hline 3 & 2525,695 & 0,0020 & 0,1 & 1331,139 & 0,527 & 1,897 & 6911,822 & 7,629 \\
\hline 4 & 2397,516 & 0,0017 & 0,2 & 1351,817 & 0,563 & 1,773 & 7733,924 & 7,489 \\
\hline
\end{tabular}

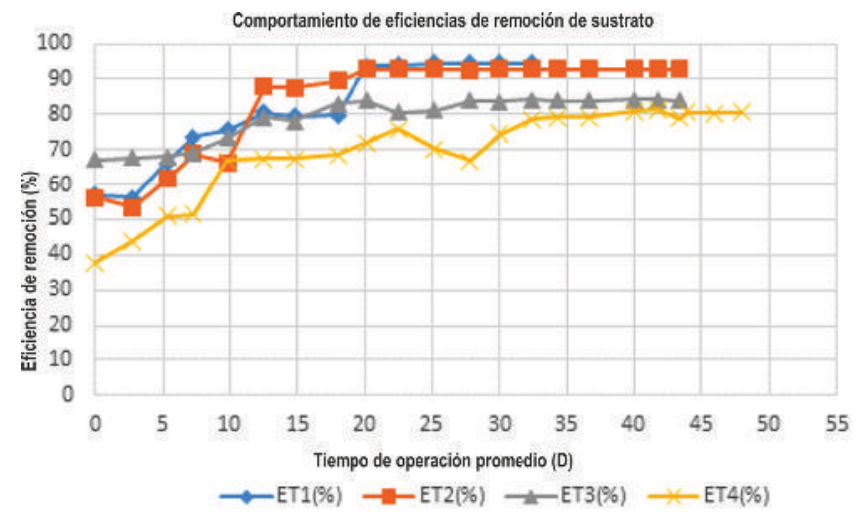

Figura 3. Comportamiento de eficiencias de remoción de sustrato

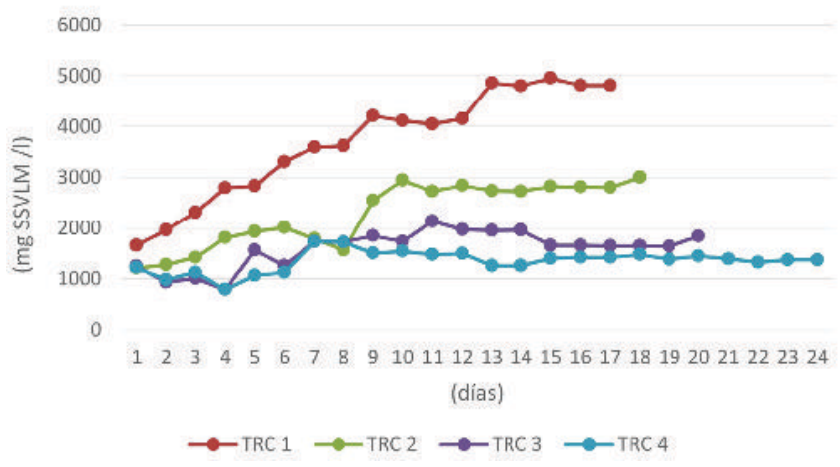

Figura 4. Comportamiento del crecimiento celular SSVLM en función a la edad del lodo TRC. 


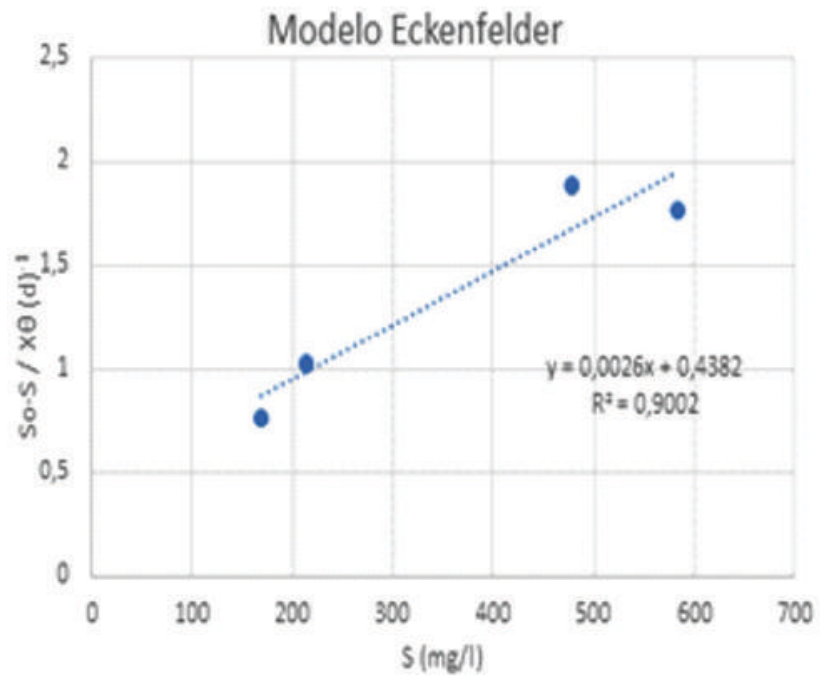

a)

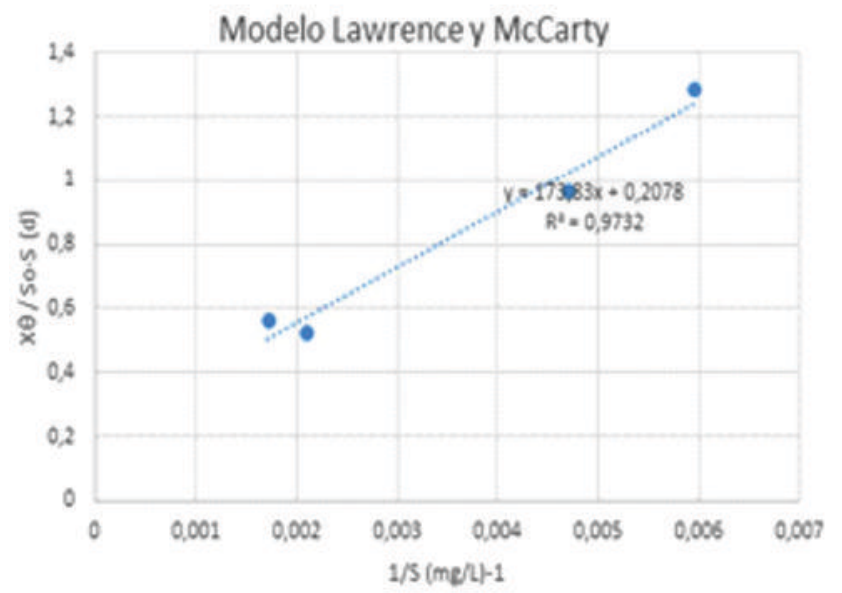

c)

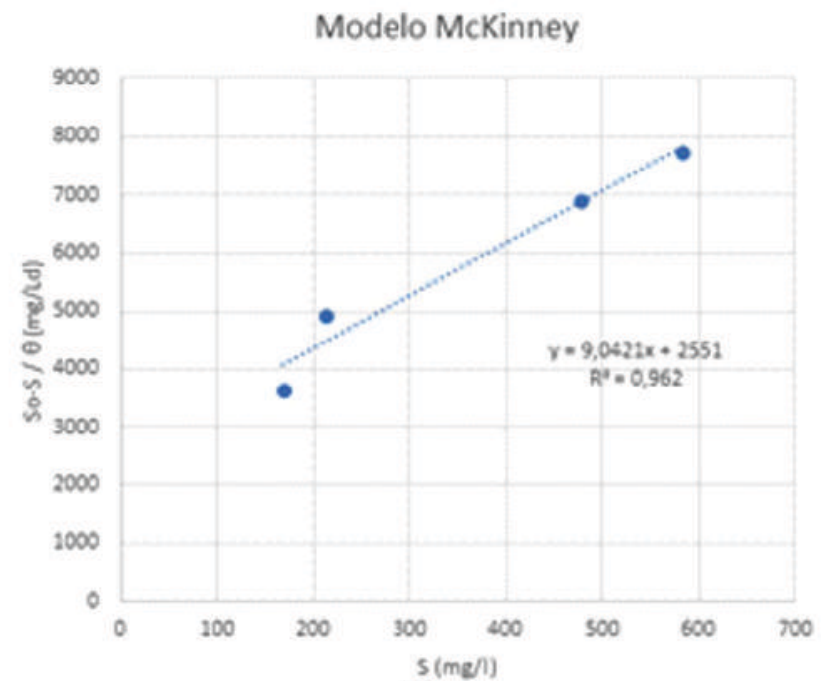

b)

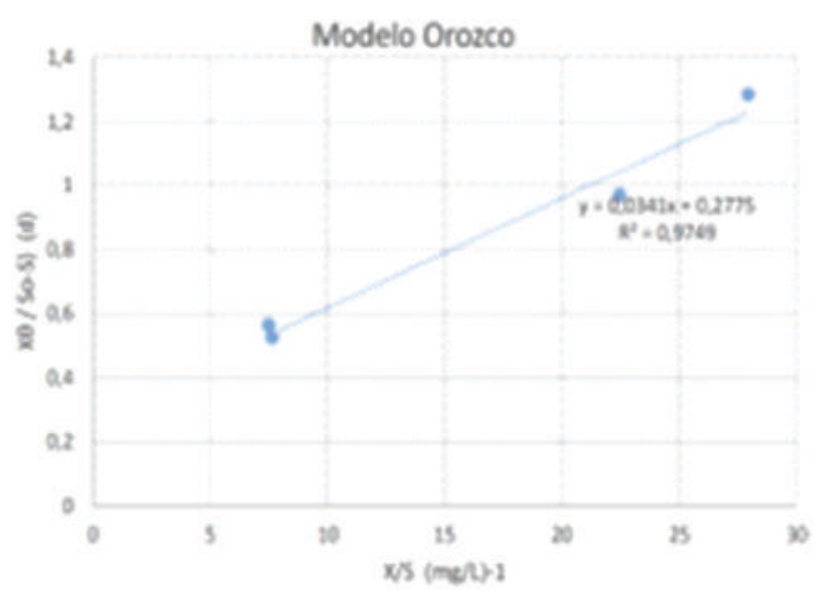

d)

Figura 5. Determinación de constantes cinéticas y estequiométricas de consumo de sustrato por regresión lineal.

A partir de los resultados obtenidos de las figuras de regresión lineal se obtuvieron las constantes cinéticas de remoción de sustrato y producción de lodo (Tabla 7).

En cuanto a la obtención de los coeficientes obtenidos experimentalmente que se presentan en la Tabla 7 y su comparación con los datos típicos para aguas residuales reportados en la Tabla 8 , indican que existe un comportamiento similar en la tasa de consumo de sustrato $(\mathrm{k})$ con un valor de $4.81 \mathrm{~d}-1$ según el modelo de Lawrence y McCarty y de $3.60 \mathrm{~d}-1$ utilizando el modelo Orozco. Situación contraria presentó la tasa de producción de biomasa cuyos valores obtenidos, $\mathrm{Y}=$ $0.089 \mathrm{mg} \mathrm{SSV} / \mathrm{mg}$ DQO y kd $=0.02 \mathrm{~d}-1$ se encuentran por debajo del rango típico para aguas residuales, lo que muestra una tasa de crecimiento celular más lenta

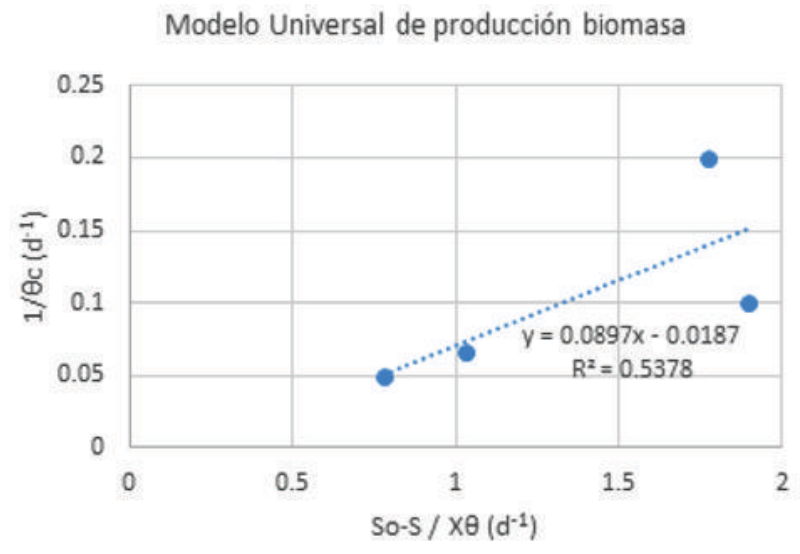

Figura 6. Determinación de constantes cinéticas y estequiométricas de producción de biomasa. 
Tabla 7. Coeficientes cinéticos y estequiométricos obtenidos experimentalmente

\begin{tabular}{|c|c|c|c|c|c|}
\hline Coeficiente & Unidad & Modelo & Ecuación & $\mathbf{R}$ & Coeficiente \\
\hline $\begin{array}{c}k \\
K L\end{array}$ & $\mathrm{mgSSV} / \mathrm{L} \mathrm{d}^{-1}$ & $\begin{array}{l}\text { Eckenfelder } \\
\text { Mc Kinney }\end{array}$ & $\begin{array}{c}y=0,0026 x+0,4382 \\
y=9,0421 x+2551\end{array}$ & $\begin{array}{c}0,9002 \\
0,962\end{array}$ & $\begin{array}{l}0,0026 \\
9,0421\end{array}$ \\
\hline $\begin{array}{c}\mathrm{k} \\
\mathrm{Km} \text { o Ks }\end{array}$ & $\begin{array}{c}\mathrm{d}^{-1} \\
\mathrm{mg} / \mathrm{L}\end{array}$ & $\begin{array}{c}\text { Lawrence y } \\
\text { McCarty }\end{array}$ & $y=173,83 x+0,2078$ & 0,9732 & $\begin{aligned} k & =4,8123 \\
K s & =836,525\end{aligned}$ \\
\hline $\begin{array}{c}\text { k } \\
\text { Ko } \\
\end{array}$ & $d-1$ & Orozco & $y=0,0341 x+0,2775$ & 0,9749 & $\begin{array}{c}k=3,6036 \\
\mathrm{Ko}=0,1228\end{array}$ \\
\hline Y Kd & $\begin{array}{c}\mathrm{mg} \mathrm{SSV} / \mathrm{mg} \text { DQO } \\
\mathrm{d}^{-1}\end{array}$ & $\begin{array}{l}\text { Orozco } \\
\text { Orozco }\end{array}$ & $y=0,0897 x+0,0179$ & 0,5378 & $\begin{aligned} Y & =0,0897 \\
K d & =0,02 d-1\end{aligned}$ \\
\hline
\end{tabular}

en el tiempo. Lo anterior se puede deber a altos niveles de nitrógeno y metales pesados que caracterizan los lixiviados y pueden ser inhibitorios o tóxicos para el proceso biológico (Corena, 2008). Por otro lado, los TRC de 5 y 10 días reportaron valores bajos de biomasa (1000-3000 mg/LSSVLM) que al ser graficados hacen que los coeficientes cinéticos sean más pequeños. Sin embargo, el valor de decaimiento $(k d)$ es inferior a la producción de lodo (Y) lo que indica que existe un equilibrio en el reactor.

Por otro lado, la constante de saturación Ks (836.5 $\mathrm{mg} / \mathrm{L})$ se encuentra muy por encima de los valores típicos para lodos activados. A pesar que hubo una notable disminución en la concentración de materia orgánica a través del proceso de tratamiento, altos valores de Ks indican que existe poca afinidad de los microorganismos para degradar el sustrato (Orozco, 2014), lo cual concuerda con la baja tasa de crecimiento celular por inhibición debida a exceso de sustrato. Cárdenas et al. (2014) reportó resultados similares en el tratamiento de aguas residuales de la industria láctea utilizando un reactor discontinuo secuencial con parámetros de operación de 24 horas y una edad del lodo de 20 días de lo cual infiere que, una cantidad de la materia orgánica es degradada y otra es arrastrada y acumulada en el lodo.

\section{Conclusiones}

El RFP de lodos activados presentó un comportamiento directamente proporcional entre la remoción de sustrato y el TRC. Los mejores resultados se obtuvieron para TRC de 15 y 20 días correspondientes a Et2 y Et1 al lograrse la estabilización del sistema en un período de operación a partir de 20 días de operación con remociones mayores a $92 \%$, por lo cual se considera un rango óptimo para la biodegradabilidad de lixiviados, rango superior al operado en aguas residuales domésticas y algunas de tipo industrial.

Para altas concentraciones de sustrato (So $\geq 2500$ $\mathrm{mg} / \mathrm{L}$ de DQO), como es el caso que se presenta en lixiviados mediante el sistema de lodos activados RFP con reciclo de lodos se comporta de acuerdo a una cinética química de primer orden, sin embargo las constantes cinéticas obtenidas son muy inferiores a las reportadas en estudios con aguas residuales domésticas e industriales con concentraciones de sustrato por debajo del coeficiente de saturación.

De acuerdo a los parámetros cinéticos estimados la ejecución del sistema de tratamiento de lixiviados puede ser descrita por el modelo de Lawrence y McCarty, por medio del cual se obtuvieron los siguientes coeficientes cinéticos: $\mathrm{KS}=836.5 \mathrm{mg} / \mathrm{L}$ DQO, $\mathrm{k}=4.8123 \mathrm{~d} 1$,

Tabla 8. Coeficientes cinéticos típicos para aguas residuales en base a DQO.

\begin{tabular}{|c|c|c|c|}
\hline Coeficiente Cinético & Unidad & Rango & Valor Típico \\
\hline k & $\mathrm{d}^{-1}$ & $2,0-10$ & 5,0 \\
\hline Km o Ks & $\mathrm{mg} / \mathrm{L}$ & $25-100$ & 60 \\
\hline $\mathrm{Y}$ & $\mathrm{mg} \mathrm{SSV} / \mathrm{mg} \mathrm{DQO}$ & $0,25-0,4$ & 0,4 \\
\hline $\mathrm{Kd}$ & $\mathrm{d}^{-1}$ & $0,04-0,075$ & 0,06 \\
\hline
\end{tabular}

Fuente. Orozco Jaramillo, A. (2014). Bioingeniería de aguas residuales. Teoría y diseño. Editorial Acodal. $2^{\circ}$ edición. Bogotá, Colombia. p 145. 
$\mathrm{Y}=0.089 \mathrm{mg}$ SSVLM $/ \mathrm{mg}$ DQO y $\mathrm{kd}=0.018 \mathrm{~d}-1$. Las mismas facilitaran proyecciones de diseño del reactor flujo pistón a escalas superiores en condiciones de temperatura similar.

\section{Agradecimientos}

A la empresa Aseo Urbano, por el aporte de análisis de laboratorios y toma de muestras.

\section{Referencias}

Álvarez CA y Suárez GJ. Tratamiento biológico del lixiviado generado en el relleno sanitario "El Guayabal" de la ciudad San José de Cúcuta. Revista Ingeniería y Desarrollo, 2006;20:95-105.

APHA-American Public Health Association. 2005. Métodos normalizados para el análisis de aguas potables y residuales. 21 Edición. APHA, AWWA, WPCF. Madrid, España.

Arévalo A, Ramón J y Ramón J. Estimación cinética de los procesos biológico de lixiviados, a partir de un sistema experimental con humedales artificiales utilizando especies nativas. Revista de Tecnología, 2015;14(2):37-48.

Arguadas A. 2013. Parámetros de control de un sistema biológico. [Fecha de Consulta 21 de Marzo de 2017]. URL: https:// es.scribd.com/document/366282826/Parametros-deControlde-Un-Sistema-Biologico.

Cárdenas C, Landeta K, Angulo N, Trujillo A, Flores P. Determination of kinetic constants for the design of biological treatment dairy effluent in batch system. Revista Técnica de la Facultad de Ingeniería . 2014;37(1):11-19.

Chávez MW. 2011. Tratamiento de lixiviados generados en el relleno sanitario de la $\mathrm{Cd}$. de Chihuahua, México: CHIHUAHUA. Tesis como requisito para obtener el grado de Maestro en Ciencia y Tecnología Ambiental.

Corena L Mironel. 2008. Sistemas de tratamientos para lixiviados generados en rellenos sanitarios. Tesis de grado. Universidad de Sucre Facultad de Ingeniería. Sincelejo, Colombia. 104p.

Espinosa-Rodríguez MA, Flores-Ánamo N, Esparza-Soto M, Fall C. Efecto de la temperatura en la tasa de crecimiento y decaimiento heterotrófico en el rango de $20-32^{\circ} \mathrm{C}$ en un proceso de lodos activados. Revista Mexicana De Ingeniería Química, 2012;11(2):309-332.

Giraldo E. Tratamiento De Lixiviados De Rellenos Sanitarios: Avances Recientes. Revista de Ingeniería Universidad de Los Andes. Departamento de Ingeniería Civil y Ambiental. Bogotá, Colombia.

Giraldo L, Restrepo I. 2003 Arranque y operación de un reactor experimental de lodos activados para el tratamiento de aguas residuales urbanas. Pregrado en Ingeniería Química. Manizales, Universidad Nacional de Colombia.

López A, Sánchez I. 2015. Postratamiento en escala piloto del lixiviado del relleno sanitario Antanas (Pasto-Nariño) por filtraciónadsorción con arena, antracita y carbón activado. Revista de Ingeniería. 2015; 43:10-15.
Metcalf y Eddy Inc. 2003. Wastewater engineering treatment, disposal and reuse. $4^{\mathrm{a}}$ ed. New York: Mc Graw Hill.

Orozco-Jaramillo A. 2014. Bioingeniería de aguas residuales. Teoría y diseño. Editorial Acodal. $2^{\circ}$ edición. Bogotá, Colombia. p 136 $-150$

Peng Z, Peng Y, Yu Z. et al. 2012. Control de la sedimentación de lodos y eliminación de nitrógeno en condiciones de bajo nivel de oxígeno disuelto. Sci. Ing. 2012;6:884-891. URL:https://doi. org/10.1007/s11783-012-0408-9

Ramírez CE. 2008. Fundamentos del proceso de lodos activados. Editorial Universitaria Universidad Nacional de México. México, 96p.

Ramón VJL, Ramón VJ, Maldonado BO. Software de simulación para el diseño de reactores de lodos activados. Revista Colombiana de Tecnologías de Avanzada. 2016;2(28):74-77.ISSN: 16927257.

Ramos VC. 2017. Evaluación de un sistema de tratamiento de lodos activados para la remoción de carga orgánica en las aguas residuales domesticas generadas en el colegio San Viator. Tesis de grado. Fundación Facultad De Ingenierías. Universidad De América Programa De Ingeniería Química. Bogotá D.C, Colombia.

Rodríguez LM, Molina FJ. Estrategias Operacionales para el Control de Problemas de Baja Sedimentación Causados por Bacterias Filamentosas en Plantas de Lodos Activados. Revista Científica en Ciencias Ambientales y Sostenibilidad (CAS). 2017;4(1):1-19.

Rodríguez R, Osorio G. 2014. Estudio de la influencia del TRC sobre la reducción de materia orgánica carbonácea y nitrógeno en una nueva configuración del sistema de estabilización por contacto tratando agua residual doméstica. Tesis de pregrado. Universidad del Valle, Santiago de Cali, Colombia. 93p.

Romero RJ. 2005. Tratamiento de aguas residuales. Teoría y principios de diseño. 3a. ed., 2a. reimp. ISBN: 9588060133. Escuela Colombiana de Ingeniería. Bogotá, Colombia. [Fecha de Consulta 21 de Agosto de 2017]. URL: https://www.libreriapensar. com/tienda/tratamiento-de-aguas-residuales-teoria-yprincipiosde-diseno-lemoine/.

Torres LP, Barba Ho L, Ojeda C, Martínez J, Castaño Y. Influencia de la edad de lixiviados sobre su composición físico-química y su potencial de toxicidad. Revista U.D.C.A Actualidad y Divulgación Científica, 2014;17(1):245-255.

Vargas RJC, Torres VJA. 2008. Diseño, implementación y estudio hidrodinámico de un reactor biológico de cargas secuenciales de flujo a pistón para tratar aguas residuales sintéticas. [Fecha de Consulta 15 de Agosto de 2016]. URL: https://ciencia.lasalle. edu.co/ing_ambiental_sanitaria/255.

Von-Sperling M. 2012. Principios del Tratamiento Biológico de Aguas Residuales. "Introducción a la calidad del agua y al tratamiento de aguas residuales". Editorial Universitaria, 1a edición en español. Vol 1. Universidad de Nariño. San Juan de Pasto. p. 468.

Rosas Jenny: https://orcid.org/0000-0001-6912-136X Ramon Jacipt: https://orcid.org/0000-0002-2030-4904 